\title{
Assessment of Urine Analysis Diagnostic Role: A Cross-Sectional Study in South Eastern of Iran
}

\author{
Mohsen R. Chenari ${ }^{1}$, Shahram Gooran ${ }^{2}$, Amin Zarghami $^{3}$, Faramarz Fazeli ${ }^{4}$ \\ ${ }^{1}$ Student Research Committee, Zahedan University of Medical Sciences, Zahedan, Iran \\ ${ }^{2}$ Urology Research Center, Sina Hospital, Tehran University of Medical Sciences, Tehran, Iran \\ ${ }^{3}$ Student Research Committee, Babol University of Medical Sciences, Babol, Iran \\ ${ }^{4}$ Department of Urology, Zahedan University of Medical Sciences, Zahedan, Iran \\ Email: *fazeli88@yahoo.com
}

Received April 30, 2012; revised August 27, 2012; accepted September 17, 2012

\begin{abstract}
Introduction: Testing for the presence of micro-organisms in the urinary tract, in order to diagnose asymptomatic bacteriuria or symptomatic urinary tract infections (UTI), is very common at all levels of health care. This study was conducted to assess the diagnostic values for bacteriuria and pyuria and evaluate their roles in therapeutic decision making. Methods: A total of 1770 urine samples were obtained by simple randomized method from the central Laboratory of the Ali-ebne-Abitaleb Hospital (Zahedan, Iran). Urine culture was done to compare urine microscopy profiles. Sensitivity, specificity, positive and negative predictive values of the findings of urine analysis were described. Results: 1055 $(59.6 \%)$ of the cases were females. 13\% (230/1770) of patients had positive urine cultures. The most prevalent cultured micro-organism was E. coli $(63 \%)$. Sensitivity, specificity, positive and negative predictive values of microscopic pyuria were $85 \%, 88 \%, 51 \%, 97 \%$, respectively. As the same for bacteriuria, these calculations were $97 \%, 98 \%, 90 \%, 99 \%$ and for the category with both bacteriuria and pyuria were $82 \%, 99 \%, 95 \%, 97 \%$, respectively. Conclusion: According to the results, it is concluded that the urine microscopy features seems to be useful to exclude the presence of infection if the results of both bacteriuria and pyuria are negative, but positive test results have to be confirmed.
\end{abstract}

Keywords: Urinary Tract Infection; Bacteriuria; Pyuria

\section{Introduction}

Urinary tract infection (UTI) refers to the presence of microbial pathogens within the urinary tract. The spectrum of disease varies from asymptomatic bacteriuria to potentially life-threatening pyelonephritis [1]. Urinary tract is sterile normally, and it could be infected by the invasion of bacteria, virus, parasite or fungus [2]. Annually about 150 million cases are reported as UTI [3]. Epidemiology is different in sex and age. The incidence is obviously more in women because of the anatomical differences in females. Except during the first few months of life, females are far more susceptible than males to UTI [4]. Bacteriuria is more common with increasing age. For elderly women living in the community, UTIs compromise the second most common infection, whereas in residents of long-term care facilities and hospitalized subjects, it is the number one cause of infection [5]. Elderly non-institutionalized women and men show a prevalence rate of $6 \%-30 \%$ and $11 \%-13 \%$, respectively, while in institutionalized cases, it ranges from

\footnotetext{
*Corresponding author.
}

$25 \%$ to $50 \%[6]$.

Recent researches improved our insight about utilities of diagnostic tests. Gold standard for diagnosis of UTI is quantitative urine culture for specific bacteria [2]. However, this procedure is costly, and takes at least 24 hours for reply; whereas an ideal test requires only limited technical skill, is cheap and has a high accuracy, enabling a quick diagnosis in high-risk patients [6].

Urine analysis (U/A) is one of the most important tests in clinical laboratories for diagnosis, screening and prevention of UTI and nowadays is used as a guide for empirical treatment in UTI $[7,8]$. Among factors, studying in U/A, leukocyte esterase, nitrite, bacteria and WBC have diagnostic value. Urine sample can be studied for leukocyte esterase in short time [2]. Leukocyte esterase and nitrite can be evaluated by using dipstick. On the other hand, presence of bacteria and WBC is important for diagnosis, screening and prevention of UTI. Presence of any bacterium in microscopic study defined as bacteriuria and presence of more than $3 \mathrm{WBC}$ in high-power field microscope defined as pyuria [9]. The important point is that U/A is quickly operable and less expensive 
[10]. Basically, U/A can be used for differential diagnosing, screening and prevention of UTI. Thus, in this study we aimed to evaluate the sensitivity, specificity, positive predictive value (PPV) and negative predictive value (NPV) for two parameters of U/A (e.g. bacteriuria, pyuria) that suggest UTI.

\section{Method and Material}

This was a prospective cross-sectional study which conducted in 2011 on 1780 urine samples that were collected from central laboratory of Ali-ebne-Abitaleb hospital in Zahedan, South-East province of Iran. The study was approved by the Research Ethics Committee of Zahedan University of Medical Sciences. Samples were collected with systematic randomized sampling method. Those referred urine samples to the laboratory in two specific days of the week during the study period, which has both urine analysis and culture results defined as inclusion criteria.

\subsection{Sample Collection}

Urine samples were obtained from cases early in the morning or several hours after having meal according to standard techniques. Urine samples were collected by the midstream clean catch method in adults and urine bag or catheterization method in children. Samples were transferred to the laboratory immediately. Samples must be cultured in two hours and if that was not possible, the urine samples were chilled in the refrigerator of 4 degrees of centigrade, awaiting for appropriate time. The samples usually were reached the laboratory and being cultured within 24 hours.

\subsection{Urine Analysis}

In the first step of microscopic evaluation, $10 \mathrm{ml}$ of urine sample was centrifuged at $2500-3000 \mathrm{rpm}$ for 5 minutes. After centrifuge supernatant was removed. Then one drop of sediment was placed onto the microscope slide, covered and examined using light microscope under 40x magnifications. Any bacteria (0 - 4) was defined as bacteriuria and leukocyte more than 3 - 5 in one high power field (hpf) was defined as pyuria.

\subsection{Urine Culture}

Urine sample was taken with calibrated sterile inoculating loops and fractioned on the surface of two plates; a blood agar base and a McConkey agar by streak method. Plates were incubated for approximately 24 hours at $35^{\circ} \mathrm{C}-37^{\circ} \mathrm{C}$. If there were no growth occurred after first time incubation they were further incubated 24 hours. Therefore, no growth after 48 hours was reported as negative. A culture with growth of potentially pathogenic bacteria was normally considered positive if the number of colony-forming units per milliliter $(\mathrm{CFU} / \mathrm{mL})$ was more than $10^{5}$. Then, grown colonies were counted, morphologically examined and identified by using biochemical tests. Type and subtype of bacteria was identified and confirmed using standard methods.

\subsection{Analysis}

In total, 1780 sample were entered in this study. The result of 10 samples were excluded because of systemic bias. For the statistical analysis the program SPSS 18 for Windows was used. Diagnostic data were analyzed using $2 \times 2$ contingency tables to calculate the sensitivity, specificity and predictive values for pyuria and bacteria, separately or in complementation which were described with the $95 \%$ Confidence Interval.

\section{Results}

Among the 1770 urine samples which were analyzed, $1055(59.6 \%)$ were adopted from female patients and $40.4 \%$ were for male patients which belonged to 6 ranges of age from less than 1 year old to more than 65 years old (Table 1). All of them have both the result of urine culture and urine microscopy. Thirteen percent (230/1770) of patients had positive urine cultures. Among the cultures with microbial growth, $64.7 \%$ (149/230) were observed in females. The prevalence of microorganisms cultured samples was: Escherichia coli $(63.5 \%$; 146/230), Enterobacter spp. (22.1\%; 51/230), Shigella spp. (4.3\%; 10/230), Proteus and Klebsiella spp. had the same prevalence $(3.5 \% ; 8 / 230)$ and the remained $3.1 \%$ were other species such as Serratia spp. and Citrobacter spp.

433 patients were positive for bacteriuria or pyuria. 383 patients were positive for pyuria which 193 cases (53.3\%) had positive urine culture. 248 patients were postive for bacteriuria which $223(89.9 \%)$ had positive urine culture. 198 patients were both positive for bacteriuria and pyuria which 189 cases (95.4\%) among them had

Table 1. UTI distribution by age.

\begin{tabular}{cccc}
\hline \multirow{2}{*}{ Age range } & \multicolumn{3}{c}{ Culture } \\
\cline { 2 - 4 } & Positive & Negative & Total \\
\hline$<1$ & $62(35.5 \%)$ & $509(28.8 \%)$ & $571(32.3 \%)$ \\
$1-5$ & $42(2.4 \%)$ & $350(19.8 \%)$ & $392(22.1 \%)$ \\
$6-15$ & $34(1.9 \%)$ & $255(14.4 \%)$ & $289(16.3 \%)$ \\
$16-35$ & $26(1.5 \%)$ & $169(9.5 \%)$ & $195(11 \%)$ \\
$36-65$ & $43(2.4 \%)$ & $160(9 \%)$ & $203(11.5 \%)$ \\
$>65$ & $23(1.3 \%)$ & $97(5.5 \%)$ & $120(6.8 \%)$ \\
Total & $230(13 \%)$ & $1540(87 \%)$ & $1770(100 \%)$ \\
\hline
\end{tabular}


positive urine culture (Table 2). The sensitivity, specificity, predictive values (positive and negative) for the parameters analyzed as predictors of UTI in this study was shown in Table 3.

\section{Discussion}

In our study most of positive results were females $(64.7 \%)$ which confirms findings of previous studies. The main reason is because of anatomical and physiological differences between two sex [11]. In this study Escherichia coli was isolated from $64.3 \%$ of cultured samples that is the most frequency among all ages. This result was approximately similar to the findings of Nys et al. who reported $66 \%$ in Netherlands [12]; but Koeijers et al. reported the rate of $48 \%$ [13]. In that study all samples were obtained from male patients who had more than 18 years old. In another study which conducted on children also Ecoli has $60 \%$ prevalence rate [14]. In our study Enterobacter and shigella were in second and third place whereas in results of two studies in Netherlands and Brazil, proteus and klebsiella were in similar rank. Maybe this variation is because of obvious discrepancy level of hygiene among two countries $[8,12]$. It must be noted that despite of low prevalence of Staphylococcus Saprophyticus, mentioning in different studies, it is an invasive microorganism often affecting the upper urinary tract, with a high probability of recurrent infections [8].

Urinalysis is a high-value procedure which requires specific labor. However, urinalysis parameters are still widely obtained to guide empirical treatment of UTI. One of the parameters which evaluated in urinalysis is pyuria. Pyuria defined as unusual presence of polymorphonuclear leukocytes in urine, indicates that an inflammatory response is occurring somewhere in the urinary tract. Although pyuria is the most prevalent manifestation of UTI; other important conditions must be considered such as: pregnancy, fever and administration of corticosteroids [8]. According to this point, history taking is so important, especially in women. In our study history was not taken and it seems the main weakness of study. Another point is that sensitivity of pyuria in detecting enterococcus and yeast infection is lower than that for gram-negative bacillus. Also this test is not reliable for confirming the diagnosis in neurogenic bladder [9].

It is well known that the sensitivity of a test is the proportion of true positive results detected by the test, while specificity is the proportion of true negatives detected [15]. According to our findings among sample population, urine microscopy on the basis of pyuria or bacteriuria was able to diagnose $82 \%$ - $97 \%$ of the patients. On the other hand, except in the testing by pyuria which had the specificity rate of $88 \%$, urine microscopy was able of $99 \%$ screening among non-patient cases. As the same for predictive values, PPV and NPV are defined as the proportion of positive and negative tests that are confirmed as detecting or excluding disease, respectively [15]. Findings of our study indicated that except in the case of pyuria which was $52 \%$ for PPV, in other conditions were calculated more than 90\%. Similarly for NPV, in all conditions it was calculates more than $97 \%$. In the study of Rehmani et al. on 984 samples in 2008, which has the similar objectives to our study, sensitivity, specificity, PPV and NPV for bacteriuria were respectively $80 \%, 83 \%, 73 \%$ and $95 \%$ and as the same for pyuria it was reported $82 \%, 81 \%, 73 \%, 95 \%$ [16]; but the findings of Yildirim et al. for pyuria among 256 cases, were reported $32 \%, 93.7 \%, 83.7 \%$ and $58 \%$ respectively. According to the low sensitivity of pyuria in their study, they suggested that pyuria as a single test, could not be able to diagnose asymptomatic bacteriuria [17]. In the study of Wiwanitkit et al. the diagnostic values of gram staining among 95 sample were all nearly $90 \%$ [18]. It should be noted that the high rate of sensitivity for bacteriuria in our study, may because of that we dedicated the cut off level of $0-4$ bacteria for positive results. As Lammers et al. demonstrated in their

Table 2. Urinalysis results.

\begin{tabular}{ccccc}
\hline \multirow{2}{*}{ Category } & & \multicolumn{2}{c}{ Urine culture } & Total \\
\cline { 2 - 4 } & & Pos. & Neg. & \\
\hline \multirow{2}{*}{ Bacteriuria } & Pos. & 222 & 26 & 248 \\
& Neg. & 8 & 1514 & 1522 \\
& Pos. & 195 & 188 & 383 \\
Pyuria & Neg. & 35 & 1352 & 1387 \\
& Pos. & 189 & 9 & 198 \\
$\begin{array}{c}\text { Bacteriuria \& } \\
\text { pyuria }\end{array}$ & Neg. & 41 & 1531 & 1572 \\
\hline
\end{tabular}

Table 3. Diagnostic values.

\begin{tabular}{ccccc}
\hline Urinalysis parameters & Sensitivity \% (95\% CI $)$ & Specificity \% (95\% CI) & PPV \% (95\% CI) & NPV \% (95\% CI) \\
\hline Bacteriuria & $97(94-97)$ & $98(98-99)$ & $90(86-93)$ & $99(99-100)$ \\
Pyuria & $85(80-89)$ & $88(86-89)$ & $51(46-56)$ & $97(97-98)$ \\
Bacteriuria \& pyuria & $82(77-87)$ & $99(99-100)$ & $95(93-98)$ & $97(97-98)$ \\
\hline
\end{tabular}


study, if the urine culture reference standard is set higher, the NPV will increase, but more numbers of infections will be missed [19]. The precision of predictive values is dependent on the sample size and use some kind of interval estimate appropriately [20]. In present study we tried to pay extra attention to this point in contrast to others with the same objectives by choosing an appropriate deal sampling size and estimating 95\% CI in reporting diagnostic values which could be better support to our findings.

In this study NPV was $97 \%$ in all cases that is strong for a diagnostic test, and bring up property of these tests in determination of urinary health. In the other word, in urinalysis if confidently there is no pyuria or bacteriuria with we could confirm that there is no UTI. This point is important for health care system as a view of cost-effectiveness. Because routinely those cultures which being ordered for confirmation of negative tests and not few in quantity, would be cut down. Therefore 1567 patients in this study did not require to be ordered for further urine culture, because of their normal urine analysis.

Moreover than exclusion of infection, establishing of that is critical in health care. The presence or absence of pyuria is not helpful in establishing the diagnosis in older patients. However, the presence of pyuria is not a valid diagnostic criterion for infection. Arinzon et al. also concluded that positive dipstick tests for LE and/or nitrite are not specific indicators of UTI, and are not suitable for screening of long-term-care inpatients for UTI because of high false negative rates of the LE and nitrite [21]. A positive urine culture and pyuria without urinary tract symptoms have been considered nondiagnostic for UTIs in this elderly nursing home population. Thus, pyuria should not influence clinical decision making about antimicrobial therapy in those with asymptomatic bacteriuria $[22,23]$. So as for nitrite and leukocyte esterase, which their results have more diagnostic if being together, the same is true for urine microscopy parameters. Deville et al. meta-analysis results, confirmed that dipstick individually, can rule out infection in population, if both nitrite and leukocyte esterase are negative. In this condition, sensitivities of the combination of both tests vary between $68 \%$ - 88\% in different populations; although positive results have to be confirmed by other methods [6].

In most studies one diagnostic test is not reliable for confirmation of UTI, so researchers consider a combination of results as the best choice for clinical decision making [8]. Microscopy is more time consuming and expensive to perform than a dipstick test, but potentially quicker and cheaper than culture. As with dipstick tests, a combination of microscopy for pyuria and bacteriuria can be used accurately to rule in and rule out a UTI. In the case of indeterminate test result, confirmatory culture is required in these patients [24]. Another study on dipsticks achieve to the same results in pregnant women [25]. The results of a meta-analysis in 2010 which comparing microscopy and urine dipstick testing by using bacterial colony count on urine culture over six studies indicated no significant difference between these methods [26].

New technological evolutions have enabled new diagnostic approaches in urinalysis such as urinary flow cytometry and automated microscopic pattern recognition [27] but such approaches needed more studies to evaluate benefits and cost-effectiveness aspects of them. Researches in this field can be improved if inclusion and exclusion criteria become explicit. Some other factors that affect accuracy are: reporting on the distribution of micro-organisms, the way in which urine is collected, the time delay between collection and analysis, the handling of mixed cultures and contaminated urine samples, and who was reading the test, may improve future studies [6].

\section{Conclusion}

According to our results it can be concluded that absence of pyuria and bacteriuria simultaneously has reliable diagnostic value for ruling out the infection; nevertheless positive results need to be confirmed by a superior method. There are numerous and different factors such as: procedure of collecting patients, sampling and performing tests, which would influence on results. Therefore, methodological quality of the studies and following standard protocols could improve the accuracy.

\section{Acknowledgements}

Financial support was obtained from the deputy of research and technology of the Zahedan University of Medical Sciences. We would like to thank the personnel of the central laboratory of the Ali-ebne-Abitaleb hospital (Zahedan, Iran). We especially appreciate to Dr. Ali Bijani for his guidance in analysis of the data.

\section{REFERENCES}

[1] E. A. Tanagho and J. W. McAninch, "Bacterial Infections Smith's General Urology," 17th Edition, McGraw-Hill Medical, New York City, 2008.

[2] H. Sorkhi, A. Jabarian and A. Asqarian, "E. Coli and It's Drug Resistance in Childhood Urinary Tract Infectious," Guilan University of Medical Sciences, Vol. 14, No. 54, 2005, pp. 8-23.

[3] M. Akram, M. Shahid and A. U. Khan, "Etiology and Antibiotic Resistance Patterns of Community-Acquired Urinary Tract Infections in JNMC Hospital Aligarh India," Annals of Clinical Microbiology and Antimicrobials, Vol. 6, No. 4, 2007, p. 4.

[4] B. Foxman, "Epidemiology of Urinary Tract Infections: 
Incidence, Morbidity, and Economic Costs," American Journal of Medicine, Vol. 113, No. S1A, 2002, pp. 5S$13 \mathrm{~S}$.

[5] S. J. Matthews and J. W. Lancaster, "Urinary Tract Infections in the Elderly Population," American Journal of Geriatr Pharmacother, Vol. 9, No. 5, 2011, pp. 286-309.

[6] W. L. Deville, J. C. Yzermans, N. P. van Duijn, et al., "The Urine Dipstick Test Useful to Rule out Infections. A Meta-Analysis of the Accuracy," BMC Urol, Vol. 4, No. 4, 2004, pp. 4.

[7] J. R. Delanghe, T. T. Kouri, A. R. Huber, et al., "The Role of Automated Urine Particle Flow Cytometry in Clinical Practice," Clinical Chimistry Acta, Vol. 301, No. 1-2, 2000, pp. 1-18.

[8] J. C. dos Santos, L. P. Weber and L. R. Perez, "Evaluation of Urinalysis Parameters to Predict Urinary-Tract Infection," Brazil Journal of Infectious Disease, Vol. 11, No. 5, 2007, pp. 479-481.

[9] T. M. Hooton, S. F. Bradley, D. D. Cardenas et al., "Diagnosis, Prevention, and Treatment of Catheter-Associated Urinary Tract Infection in Adults: 2009 International Clinical Practice Guidelines from the Infectious Diseases Society of America," Clinical Infectious Disease, Vol. 50, No. 5, 2010, pp. 625-663. doi:10.1086/650482

[10] M. Ayazi and M. Daneshi, "Comparison of Urine Culture and Urine Dipstick Analysis in Diagnosis of Urinary Tract Infection," Acta Medical Iranica, Vol. 45, No. 6, 2007, pp. 501-504.

[11] S. P. McLaughlin and C. C. Carson, "Urinary Tract Infections in Women," Medical Clinics of North America, Vol. 88, No. 2, 2004, pp. 417-429.

[12] S. Nys, T. van Merode, A. I. Bartelds and E. E. Stobberingh, "Urinary Tract Infections in General Practice Patients: Diagnostic Tests versus Bacteriological Culture," Journal of Antimicrob Chemother, Vol. 57, No. 5, 2006, pp. 955-958.

[13] J. J. Koeijers, A. G. Kessels, S. Nys, A. Bartelds, G. Donker, E. E. Stobberingh and A. Verbon, "Evaluation of the Nitrite and Leukocyte Esterase Activity Tests for the Diagnosis of Acute Symptomatic Urinary Tract Infection in Men," Clinical Infectious Disease, Vol. 45, No. 7, 2007, pp. 894-896.

[14] S. Antwi, I. Bates, B. Baffoe-Bonnie and J. Critchley, "Urine Dipstick as a Screening Test for Urinary Tract Infection," Annals of Tropical Paediatrics, Vol. 28, No. 2, 2008, pp. 117-122. doi:10.1179/146532808X302134

[15] H. Kelly, A. Bull, P. Russo and E. S. McBryde, "Estimating Sensitivity and Specificity from Positive Predictive Value, Negative Predictive Value and Prevalence: Application to Surveillance Systems for Hospital Acquired Infections," Journal of Hospital Infection, Vol. 69, No. 2, 2008, pp. 164-168.

[16] R. Rehmani, "Comparison of Urine Dipstick, Microscopy and Culture for Detection of Bacteriuria in Childhood," Clinical Pediatrics, Vol. 29, No. 4, 2009, pp. 214-218.

[17] M. Yildirim, I. Shahin and A. Kucukbayark, "The Validity of the Rapidly Diagnostic Tests for Early Detection of Urinary Tract Infection," Duzcetip Fakultesi Dergisi, Vol. 3, 2008, pp. 39-42.

[18] V. Wiwanitkit, N. Udomsantisuk and C. Boonchalermvichian, "Diagnostic Value and Cost Utility Analysis for Urine Gram Stain and Urine Microscopic Examination as Screening Tests for Urinary Tract Infection," Urological Research, Vol. 33, No. 3, 2005, pp. 220-222. doi:10.1007/s00240-004-0457-z

[19] R. L. Lammers, S. Gibson, D. Kovacs, W. Sears and G. Strachan, "Comparison of Test Characteristics of Urine Dipstick and Urinalysis at Various Test Cut off Points," Annals of Emergency Medicine, Vol. 38, No. 5, 2001, pp. 505-512.

[20] P. D. Sundvall and R. K. Gunnarsson, "Evaluation of Dipstick Analysis Among Elderly Residents to Detect Bacteriuria: A Cross-Sectional Study in 32 Nursing Homes," BMC Geriatrics, Vol. 9, No. 32, 2009, p. 32.

[21] Z. Arinzon, A. Peisakh, I. Shuval, S. Shabat and Y. N. Berner, "Detection of Urinary Tract Infection (UTI) in Long-Term Care Setting: Is the Multireagent Strip an Adequate Diagnostic Tool?" Arch Gerontol Geriatrics, Vol. 48, No. 2, 2009, pp. 227-231.

[22] L. E. Nicolle, "Urinary Tract Infections in Longterm Care Facilities," Infection Control and Hospital Epidemiology, Vol. 22, No. 3, 2001, pp. 167-175.

[23] L. E. Nicolle, "Asymptomatic Bacteriuria: Review and Discussion of the IDSA Guidelines," International Journal of Antimicrob Agents, Vol. 28, No. S1, 2006, pp. S42S48.

[24] P. Whiting, M. Westwood, L. Bojke, et al., "Clinical Effectiveness and Cost-Effectiveness of Tests for the Diagnosis and Investigation of Urinary Tract Infection in Children: A Systematic Review and Economic Model," Health Technological Assess, Vol. 10, No. 36, 2006, pp. $1-154$.

[25] J. O. Eigbefoh, P. Isabu, E. Okpere and J. Abebe, "The Diagnostic Accuracy of the Rapid Dipstick Test to Predict Asymptomatic Urinary Tract Infection of Pregnancy," Journal of Obstetrics \& Gynaecology, Vol. 28, No. 5, 2008, pp. 490-495.

[26] R. Mori, N. Yonemoto, A. Fitzgerald, K. Tullus, K. Verrier-Jones and M. Lakhanpaul, "Diagnostic Performance of Urine Dipstick Testing in Children with Suspected UTI: A Systematic Review of Relationship with Age and Comparison with Microscopy," Acta Paediatria, Vol. 99, No. 4, 2010, pp. 581-584.

[27] J. Delanghe, "New Screening Diagnostic Techniques in Urinalysis," Acta Clinica Belgica, Vol. 62, No. 3, 2007, pp. 155-161. 